\title{
Apprentissage d'une pratique instrumentale en orchestre à l'école : une approche didactique
}

The training orchestra in primary school : a didactic study of teaching a musical instrument

Dominique Forest et Pascale Batézat-Batellier

\section{OpenEdition}

\section{Journals}

Édition électronique

URL : https://journals.openedition.org/educationdidactique/1812

DOI : 10.4000/educationdidactique.1812

ISSN : 2111-4838

\section{Éditeur}

Presses universitaires de Rennes

\section{Édition imprimée}

Date de publication : 31 décembre 2013

Pagination : 79-96

ISBN : 978-2-7535-3327-1

ISSN : 1956-3485

\section{Référence électronique}

Dominique Forest et Pascale Batézat-Batellier, « Apprentissage d'une pratique instrumentale en orchestre à l'école : une approche didactique », Éducation et didactique [En ligne], 7-3 | 2013, mis en ligne le 31 décembre 2015, consulté le 17 août 2022. URL : http://journals.openedition.org/ educationdidactique/1812; DOI : https://doi.org/10.4000/educationdidactique.1812 


\title{
APPRENTISSAGE D'UNE PRATIQUE INSTRUMENTALE EN ORCHESTRE À L'ÉCOLE : UNE APPROCHE DIDACTIQUE
}

\author{
Dominique Forest, CREAD, IUFM de Bretagne, Université de Bretagne Occidentale
} Pascale Batézat-Batellier, CREAD, CRR musique, danse, théâtre de Rennes

Larticle porte sur l'enseignement de la pratique d'un instrument à vent dans un orchestre, dans une école élémentaire, par des enseignants d'un Conservatoire. Il se propose de donner à voir et à comprendre cette pratique spécifique, dans ses dimensions verbales et non-verbales, et dans son « épaisseur » épistémique.

Les données recueillies sont essentiellement constituées de films vidéo. Elles sont abordées avec une perspective anthropologique dans le cadre d'une théorie de l'action conjointe en didactique et d'une méthodologie associée qui sont décrites en première partie. Sont précisés en particulier l'usage des concepts de contrat, de milieu, et d'équilibration didactique.

Quatre extraits de séances sont ensuite étudiés de façon détaillée. L'analyse met en évidence les problèmes didactiques posés par les caractéristiques sonore et corporelle du milieu, ainsi que la sémiose spécifique qui en résulte. Nous montrons comment ce processus sémiotique peut être vu comme un processus d'équilibration didactique, entre contrat et milieu.

Ces questions sont ensuite soumises à une discussion qui élargit en conclusion le propos aux dimensions sociales de la pratique.

Mots-clés : instrument de musique, orchestre, contrat, milieu, sémiose, équilibration didactique.

The training orchestra in primary school : a didactic study of teaching a musical instrument

This paper is based on an instructional sequence of music designed for primary schools. Pupils (aged 8 to 10) learn how to play a musical instrument of the brass family, in three primary schools during school time. Art Teachers come from the Regional Music School in Rennes (France).

We give a typical example of an analysis of teaching a musical instrument in this context of a training orchestra. We propose a detailed description of the situation based on the Joint Action Theory in Didactic, with the concepts of Contract and Milieu. Choosing some moments, we analyse them considering the importance of language, body and space positioning as means of supporting teacher and pupils' joint action. We argue that the teacher's work and the pupils' feedback can be viewed as a work of equilibration between the didactic contract and the didactic milieu, in a semiosis process.

Discussing the results of the analysis, we examine the following questions : what skills are taught to students learning to play an instrument within the context of an orchestra in primary school? How do they acquire this knowledge from the joint teacher / student action? To what extent can this knowledge help students learn the skills necessary to "work together» in the school and outside?

Keywords: Joint Action Theory in Didactics (JATD), music, learning, teaching, didactic equilibration, musical instrument training, orchestra. 


\section{INTRODUCTION}

Il s'agit dans cet article de donner à voir et à comprendre une pratique didactique à la fois originale dans sa démarche et spécifique dans son contenu. Notre objet d'étude est en effet l'enseignement de la pratique d'un instrument à vent dans un orchestre, dans une école élémentaire, par des enseignants d'un Conservatoire à Rayonnement Régional (CRR).

Nous plaçons notre propos dans une perspective anthropologique (Chevallard, 1992). Il s'appuie sur la Théorie de l'Action Conjointe en Didactique (Sensevy \& Mercier, 2007, Sensevy, 2011), et il a pour but d'alimenter la réflexion autour de deux questions. Tout d'abord, nous souhaitons soumettre la théorie à l'épreuve d'une pratique dont les dimensions à la fois sonores (musicales), techniques (instrumentales) et collectives (orchestre) sont susceptibles d'en tester, d'une certaine façon, la pertinence et la portée. Ensuite et dans le même mouvement, il s'agit de rendre compte de cette pratique dans sa spécificité et dans son « épaisseur » culturelle, en décrivant les comportements des professeurs et des élèves, au sein de leur action conjointe en situation, pour en identifier la cohérence et les raisons d'être.

Après une première partie théorique et méthodologique, nous centrerons notre étude sur une séance, située dans un dispositif que nous aurons décrit. Nous présenterons ensuite de façon détaillée l'analyse de quatre extraits issus de cette séance, dont les résultats seront soumis à la discussion autour des questions posées.

\section{ÉLÉMENTS THÉORIQUES ET MÉTHODOLOGIQUES}

Le « jeu » comme système de description

La théorie évoquée en introduction et que nous nommerons ci-après TACD repose sur la triple déclinaison d'un modèle, celui du jeu, en termes de jeu didactique, jeu d'apprentissage et jeu épistémique (Sensevy, 2011, 2012 ; Gruson, Forest \& Loquet, 2012)

Le jeu didactique, dans un premier niveau de description, décrit la grammaire de l'action, le mot grammaire étant entendu au sens de Wittgenstein comme structure fondamentale, ou logique propre (Chauviré \& Sackur, 2003). Dans le jeu didactique, l'un des joueurs, que nous nommerons $\mathrm{P}$, gagne si et seulement si un autre joueur, que nous nommerons $\mathrm{E}^{\mathrm{l}}$ gagne, c'est-à-dire si $\mathrm{E}$ produit les comportements attendus par P. La grammaire de ce jeu inclut une contrainte paradoxale (Brousseau, 1998) car $\mathrm{P}$ ne peut produire ce comportement à la place de $\mathrm{E}$ : tout en ne sachant pas ce qu'il faut faire exactement, E doit produire ce comportement de son propre chef. Le professeur est donc pris dans une dialectique entre réticence et expression (Sensevy \& Quilio, 2002, Sensevy, 2011) ; c'est-à-dire qu'il doit se retenir de dire ce qu'il sait (réticence) tout en produisant un certain nombre de signes pour orienter l'action de l'élève (expression). Dans l'exemple que nous traitons, le jeu didactique porte sur la pratique d'orchestre, et le gain du professeur repose au final sur la production collective par les élèves de la forme musicale attendue avec divers instruments, ce qui suppose la résolution d'un certain nombre de problèmes.

Pour gagner à ce jeu, le professeur va engager les élèves dans une succession de jeux d'apprentissage, deuxième niveau de description du modèle, qui sont déterminés par la confrontation des élèves à un certain " milieu » sous un certain « contrat " (Brousseau, 1998, Sensevy \& Mercier, op. cit., Sensevy, 2011). Décrire le milieu consistera à identifier les éléments matériels et symboliques qui vont nourrir l'action de l'élève, ou résister à cette action. Décrire le contrat consistera à expliciter les attentes réciproques du professeur et des élèves, système d'attentes constitué dans ses dimensions tant génériques que spécifiques au savoir visé, sur le fond des habitudes de la classe, du professeur et des élèves dans des situations antérieures.

Le contrat, issu de l'action antérieure, représente ainsi ce qu'il y a à faire et les moyens disponibles pour le faire, autrement dit le système stratégique actuel disponible aux élèves. Le milieu représente ce sur quoi ou avec quoi les élèves vont faire, et ce milieu est censé être porteur, dans ses potentialités et ses affordances, d'un système stratégique virtuel, celui que les élèves devront mettre en ouvre pour gagner au jeu d'apprentissage. Les changements de milieu et de contrat permettent d'identifier les changements de jeu d'apprentissage, la modélisation en termes de jeu permettant à chaque fois d'en expliciter les règles définitoires (celles qui sont nécessaires pour jouer le jeu), les règles stratégiques (celles qui permettent de gagner), et de décrire les stratégies effectivement mises en œuvre par les élèves. Nous 
Dominique Forest et Pascale Batézat-Batellier

verrons que dans le cas de la musique d'orchestre, le milieu présente des spécificités qui vont conduire à des stratégies professorales tout aussi spécifiques.

Au sein de chacun de ces jeux d'apprentissage, émergent ce que nous décrivons à un troisième niveau de modélisation comme des jeux épistémiques. Ils sont conçus comme des systèmes de capacités dont les élèves devront se rendre suffisamment familiers pour gagner au jeu d'apprentissage correspondant. Ces systèmes de capacités, qui émergent dans l'action conjointe, pourront être appréciés à l'aune de ce que la théorie désigne comme des jeux épistémiques sources, où les systèmes de capacités sont consubstantiels à des ouvres (Chevallard, 1996) telles qu'elles sont connues et pratiquées par ceux qui sont considérés comme les experts du domaine (ici, par des musiciens d'un orchestre d'harmonie). Une description exhaustive du « jeu des musiciens en orchestre » en jeu épistémique source dans toutes ses dimensions ne peut être menée ici, et seuls certains aspects en seront évoqués lors de la description des jeux d'apprentissage. On y retrouvera toutefois certains enjeux et contraintes très proches de celles de l'action conjointe d'un chef et de ses musiciens.

Par l'usage de ce modèle, nous cherchons à respecter la dynamique du processus didactique, milieu et contrat étant produits continûment, comme nous le verrons, dans le cours de l'action conjointe professeur-élève. Le modèle permet également de référer, sans toutefois l'y réduire, cette dynamique à un savoir qui n'est pas vu comme une essence mais comme une pratique désignée en anglais comme celle des « connoisseurs».

\section{Méthode et représentation de l'action}

Les données qui servent de support à cet article sont extraites d'un ensemble recueilli au cours d'un mémoire de deuxième année de master (BatézatBatellier, 2012), dans une démarche de type ethnographique et clinique.

La démarche est ethnographique, au sens où elle prend comme objet l'action elle-même, telle qu'elle se produit in situ, sans que cette action soit informée a priori par une démarche expérimentale ou une ingénierie, c'est-à-dire que cette action n'est pas le résultat d'une sollicitation du chercheur. Nous avons utilisé pour cela des films vidéos, dont nous savons qu'ils ne représentent pas la totalité objective de l'action, mais qui partagent avec celle-ci un certain nombre de caractéristiques, en particulier sémiotiques. La représentation par le film d'une action est en effet, d'une certaine façon, analogue ${ }^{2}$ à l'action, et au contraire d'un journal de bord ou d'un système de codage par catégories dans une grille d'observation, le film conserve la spécificité des agencements corporels, matériels et spatiaux.

La démarche s'inscrit également dans une clinique du didactique (Leutenegger, 2000), au sens ou il s'agit d'une démarche à la fois ascendante et attentive aux détails, dans une acception proche de celle de Foucault (1963) concernant la clinique médicale, et de celle de Ginzburg (1987) proposant un paradigme indiciaire. L'usage du film est ici également adapté à une telle analyse par les multiples manipulations qu'il permet, telles que visionnement multiple, arrêt sur image, analyse "à la sourde », etc.

La description de l'action suppose également un système de représentation de cette action, permettant à la fois de la réduire, de l'objectiver et d'en partager les analyses. Nous utilisons pour cela les ressources offertes par une mise en vision synoptique (Wittgenstein, in Glock, 2003, p. 584-591) sous forme de tableaux à différentes échelles de temps, macro, meso et micro-didactique, et qui permettent ainsi de rendre visible de façon contiguë des éléments d'action situés à différents moments, voir à différents endroits. Nous utilisons également des techniques de mise en intrigue, (Veyne, 1971), récits résumés permettant de rendre la tension autour de ce que nous avons identifié, lors de l'analyse en jeux d'apprentissages, comme étant les enjeux de l'action didactique conjointe ${ }^{3}$.

Confrontés à la nécessité d'analyser et de représenter dans cet article la part analogique de l'action, nous sommes amenés à associer photogrammes, transcription et description (Forest, 2006, Sensevy op. cit.), pour une « traduction» qui respecte autant qu'il est possible la nature des phénomènes étudiés.

\section{La question sémiotique, et l'équilibration didactique}

Cette « écriture » de l'action, en particulier dans ses dimensions meso- et micro didactiques, nécessite de pouvoir prendre en compte tous ses éléments, tant verbaux que non-verbaux ${ }^{4}$. L'importance de cette multimodalité a déjà été mise en évidence 
dans d'autres travaux d'orientations variées (voir par exemple Mondada, 2004 ; Fillietaz, 2005). Dans le cadre de la TACD, nous considérons que les processus de transmission de savoirs peuvent être vus plus généralement comme des processus de sémiose, c'est-à-dire comme production et déchiffrement de signes (Pierce, 1998 ; Sensevy, 2011 ; Sensevy \& Forest, 2012).

Dans ce que nous modélisons comme un jeu d'apprentissage, il s'agit, pour l'élève, de produire et déchiffrer des signes de nature variée afin d'appréhender la portée épistémique du jeu. Le professeur, quant à lui, joue de la réticence-expression constitutive de tout jeu didactique. Nous appelons sémiose du milieu cette première production-déchiffrement de signes, qui ne sont pas a priori intentionnels, c'est-àdire qu'ils ne relèvent pas directement d'une intention du professeur.

Cette première sémiose s'inscrit nécessairement sur le fond de l'action conjointe précédente, autrement dit dans le contrat didactique qui a permis à l'élève, a minima, de se confronter au problème posé. C'est en s'appuyant sur ce fond existant que le professeur pourra orienter l'action de l'élève. Ce processus d'orientation de l'action dans le milieu réfère à une deuxième sémiose, la sémiose du contrat.

Une caractéristique des transactions didactiques consisterait ainsi dans la recherche d'une sorte d'équilibre entre contrat et milieu dans les jeux d'apprentissage. Sensevy (2011) propose d'en rendre compte sous le terme d'équilibration didactique, en redéfinissant ce modèle d'origine piagétienne de la façon suivante : lorsque l'élève aborde une situation, il va la considérer en essayant de l'assimiler à une situation déjà rencontrée, autrement dit de la considérer en rapport à un contrat didactique existant. Cette part de l'assimilation du nouveau à l'ancien, c'est-à-dire du milieu par le contrat, ne peut être nulle, car cela signifierait que l'élève est totalement incapable de produire-déchiffrer quelque signe que ce soit dans la situation. Mais elle ne peut non plus être totale, car un apprentissage effectif suppose que l'élève, à partir de ce qu'il connaît, puisse acquérir de nouvelles capacités en se confrontant au milieu, autrement dit que soit produite dans l'action conjointe une accommodation de l'ancien par le nouveau, c'est-à-dire du contrat par le milieu.

C'est cette dialectique du nouveau et de l'ancien, de l'assimilation du milieu par le contrat et de l'accommodation du contrat par le milieu que nous nous proposons de mettre en évidence à travers les processus de sémiose dans les exemples étudiés. Mais la question sémiotique dans le cas de l'enseignement de la musique, comme nous le verrons dans les exemples empiriques, apparaît relativement complexe, tant pour le professeur que pour le chercheur.

Le professeur cherche en effet à orienter l'action des élèves, en leur donnant des indications à propos des sons qu'ils produisent. Mais il doit spécifier cette orientation à deux caractéristiques spécifiques de la sémiose du milieu

i) l'orientation porte sur un son qui est en train d'être produit, quelquefois collectivement, et il ne lui est pas possible d'utiliser les modalités sonores (verbales) au risque de perturber, voire de couvrir la production en cours

ii) il doit orienter l'action des élèves en s'appuyant sur des signes du milieu, à savoir des sons, invisibles et volatiles par nature.

Le chercheur quant à lui a pour objectif de mettre en évidence cette double sémiose du professeur, ce qui nécessite tout à la fois de représenter les différentes catégories de signes qui adviennent simultanément dans la situation, sachant que cette représentation, dans le cas des sons produits, ne peut passer que par une explicitation. Les systèmes hybrides composés de photogrammes, verbatim et commentaires trouvent peut-être ici leur limite : il conviendra à la lecture d'apprécier la nécessité de recourir à des nouvelles formes d'écritures scientifiques, hybrides multi-media, telles que permises par les nouveaux supports de publication en ligne.

\section{PRÉSENTATION DE LA SÉANCE ÉTUdiÉE}

\section{La séance dans son contexte}

La recherche porte sur un dispositif appelé " orchestre à l'école », qui fait intervenir des enseignants instrumentistes d'un conservatoire dans une école primaire. Un orchestre est formé et des instruments (le plus souvent à vent) sont mis à la disposition des élèves qui apprennent ainsi, à partir d'une pratique collective, à jouer d'un instrument. Deux classes de ce type situées dans la même agglomération urbaine fonctionnent auprès d'élèves de CE2 et de CM1 (8-9 ans, $3^{\mathrm{e}}$ et $4^{\mathrm{e}}$ primaire), l'une depuis 2010 et l'autre depuis 2011. Les deux écoles choisies 
pour l'étude sont situées dans des quartiers identifiés comme "zone d'éducation prioritaire », avec une population plutôt défavorisée, en grande majorité d'origine étrangère. Les instruments pratiqués sont dans la première école : le trombone, la trompette, le tuba, la percussion et dans la deuxième : la flûte, la clarinette et le saxo.

La séance qui supporte les analyses est extraite d'un recueil de données effectué en 2011-2012, auprès d'une classe nouvellement entrée dans le dispositif. Les leçons d'apprentissage de la musique en orchestre ont lieu dans une salle de l'école, tous les mardis matin dès le début de journée, et les professeurs des classes concernées participent à tour de rôle aux répétitions. Les élèves ont commencé à prendre leur instrument à la deuxième séance. La séance observée est donc la quatrième pour ce qui concerne le travail collectif, mais la troisième pour l'apprentissage des instruments proprement dits. Il s'agit d'une séance de révision et d'approfondissement des cours précédents, et elle inclut la participation de deux professeurs, le professeur chef d'orchestre (musicien du conservatoire titulaire d'un Diplôme d'État, ci-après nommé Pg), et un intervenant spécialisé (titulaire du Diplôme Universitaire de Musicien Intervenant, ci-après nommé Ps).

La séance débute quand les élèves ont pris leurs instruments et sont placés. Après que Pg (le professeur d'orchestre) ait rappelé le concert à venir, les instruments sont posés et Ps (l'intervenant spécialisé) commence par un échauffement corporel et vocal, pour une durée d'environ 10 minutes. Les élèves reprennent ensuite leur instrument et commencent la répétition avec Pg qui les fait travailler d'abord ensemble, puis par pupitre, puis individuellement. Cette alternance de modalités se retrouve dans toute cette séance, et également dans d'autres séances comparables. Cet enchaînement peut en outre être très rapide, et signifié par des gestes et des regards attribués, que les élèves semblent très rapidement comprendre. Dans la séance que nous allons maintenant détailler plus précisément, les élèves révisent le «Do », le « Ré », mais c'est lors de la production du « Mi » que des difficultés apparaissent.

\section{Une analyse didactique de la séance}

Une première description synthétique, au niveau $\mathrm{du}$ jeu didactique, peut s'exprimer comme suit : le professeur cherche à faire produire, par les élèves et avec des instruments différents, des sons qui sont nommés « notes » et qui doivent, simultanément, sonner juste ${ }^{5}$ et être jouées ensemble.

Le modèle nous permet ensuite de produire une description plus fine, de niveau meso-didactique, sous la forme du tableau d'analyse ci-dessous. Cette description nous permet de spécifier le jeu didactique en une succession, souvent très rapide, de jeux d'apprentissage dont le tableau représente une typologie. Nous mettons ainsi en évidence la mesogenèse (l'actualisation de différents milieux) et la topogenèse (l'évolution des places respectives du professeur et des élèves) en fonction de l'avancée du temps didactique (chronogenèse), c'est-à-dire en termes de contrat et de milieu dans un processus. On observe que le professeur et les élèves tirent parti dans leur action conjointe, au sein de chacun de ces types de jeux, des capacités étudiées et mises en œuvre lors des jeux d'apprentissage précédents.

Dans ces jeux, le contrat initial provient du système des connaissances, produites en particulier dans l'action conjointe antérieure (jeu d'apprentissages de type 1 et 2 du tableau), avec lequel l'élève va faire face au problème qui consiste à jouer une note, c'est-à-dire avoir un bon ancrage au sol, savoir souffler dans son instrument par l'embouchure, savoir reconnaître la note demandée. Ce contrat permet d'assimiler une part du milieu constituée par le sol, le corps de l'élève et le corps du professeur, l'instrument et les sons produits par soi.

Lorsque le professeur demande de jouer une note, par exemple " $\mathrm{mi} »$, il s'attend donc à ce que les élèves sachent se positionner, sachent souffler dans leur embouchure et sachent nommer les sons appris, et les élèves s'attendent à ce que le professeur leur donne les indications de la même façon que dans le contrat précédent, sur la façon de s'y prendre (sémiose du contrat didactique). Les indications sont souvent non-verbales car l'écoute des sons produits est indispensable à l'ajustement du contrat.

Dans les moments correspondant à la phase $3 \mathrm{du}$ tableau, l'attente du professeur est que chaque élève produise une note juste et constante. Or, le professeur estime souvent que le son produit par les élèves n'est pas satisfaisant. Il s'ensuit la nécessité d'une accommodation du contrat initial (produire un son stable avec une posture adéquate) car si l'assimilation précédente du milieu a bien produit un meilleur ancrage au sol, cet ancrage n'est pas suffisant pour 
répondre à l'enjeu générique : jouer un son stable et ensemble $e^{6}$. Vient alors s'ajouter, un deuxième type de contrat: "fais ce que fait mon corps (bouche, lèvres) et produis le son que je produis ». Le milieu de la première phase, qui était le corps du professeur, le corps de l'élève et le sol, est ainsi modifié : s'y ajoutent d'une part la bouche, les lèvres, l'air et le son du professeur, et d'autre part l'ouie, la bouche, les lèvres et le son produit par l'élève.

L'analyse suivante de quatre jeux d'apprentissages, issus des types $3,4,5$ et 6 du tableau, a pour but de montrer comment sont produites des représentations du son par des signes visuels, comment à partir de ces représentations visuelles le contrat

Tableau 1 : modélisation en jeux d'apprentissage de l'action conjointe visant à la production par les élèves d'une note juste ensemble

\begin{tabular}{|c|c|c|}
\hline $\begin{array}{l}\text { Succession de Jeux d'apprentissages. } \\
\text { L'enjeu générique est la production de } \\
\text { notes justes et ensemble }\end{array}$ & $\begin{array}{l}\text { Structure du milieu. } \\
\text { Les « objets » du milieu, qui appellent à } \\
\text { certaines stratégies virtuelles (« ce sur quoi/ } \\
\text { avec quoi on agit ») }\end{array}$ & $\begin{array}{l}\text { Pragmatique du contrat. } \\
\text { Les règles d'action actuelles sur les } \\
\text { « objets » du milieu (« qu'est-ce qu'il faut } \\
\text { faire?») }\end{array}$ \\
\hline $\begin{array}{l}\text { 1) assurer la stabilité corporelle: } \\
\text { « enracinement au sol » grâce à diffé- } \\
\text { rentes positions }\end{array}$ & $\begin{array}{l}\text { Corps du professeur, corps des élèves, le sol } \\
\text { et les sensations des élèves. }\end{array}$ & Contrat d'imitation « fais ce que je fais » \\
\hline $\begin{array}{l}\text { 2) travailler individuellement par } \\
\text { instrument; prendre comme exemple } \\
\text { l'exemple auditif }\end{array}$ & $\begin{array}{l}\text { Au milieu précédent s'ajoute, le son produit, } \\
\text { par le corps de l'élève et / ou celui du profes- } \\
\text { seur avec une sollicitation + importante de } \\
\text { l'ouie. }\end{array}$ & $\begin{array}{l}\text { Le contrat d'imitation évolue : produire le } \\
\text { son proposé par le professeur. }\end{array}$ \\
\hline $\begin{array}{l}\text { 3) identifier la perception auditive à la } \\
\text { perception du geste. }\end{array}$ & $\begin{array}{l}\text { Le milieu sonore trouve une représentation } \\
\text { dans le corps du professeur par les mimes } \\
\text { qu'il produit de ce qu'il attend des élèves } \\
\text { à partir de ce qu'il entend des sons qu'ils } \\
\text { produisent. L'action conjointe profes- } \\
\text { seur/élève construit donc ce milieu qui } \\
\text { comprend : la bouche, les lèvres, l'air et le } \\
\text { son du professeur et d'autre part: l'ouie la } \\
\text { bouche, les lèvres et le son des élèves. }\end{array}$ & $\begin{array}{l}\text { Contrat d'expérimentation : essai /erreur } \\
\text { /comparaison } \\
\text { «imite ce que je fais et rectifie au fur et à } \\
\text { mesure de ce que tu entends et de ce que } \\
\text { tu vois» }\end{array}$ \\
\hline $\begin{array}{l}\text { 4) jouer ensemble une même note, } \\
\text { en rectifier la justesse et la stabilité à } \\
\text { partir des indications mimo-gestuelles } \\
\text { du professeur - chef d'orchestre }\end{array}$ & $\begin{array}{l}\text { Le milieu est un « milieu-soi » Le corps est } \\
\text { toujours convoqué comme milieu, mais ce } \\
\text { sur quoi il faut agir est précisé. Les gestes de } \\
\text { la main symbolisent le son et représentent } \\
\text { une analogie du milieu : à une perception } \\
\text { auditive correspond un geste. À une rectifica- } \\
\text { tion demandée correspond une modification } \\
\text { du geste. } \\
\text { Le milieu sonore n'étant pas suffisant, il solli- } \\
\text { cite et associe deux sens : l'ouie et la vue. }\end{array}$ & $\begin{array}{l}\text { Contrat d'imitation métaphorique corpo- } \\
\text { relle : «fais techniquement ce que je te } \\
\text { montre avec mes gestes, en regardant } \\
\text { et en imitant avec ta bouche ces signes } \\
\text { nouveaux». }\end{array}$ \\
\hline $\begin{array}{l}\text { 5) ajuster le son, la justesse, la } \\
\text { technique en fonction du geste du } \\
\text { professeur-instrumentiste }\end{array}$ & $\begin{array}{l}\text { Le milieu est un milieu-soi : } \\
\text { - Du côté du professeur, le corps sert à } \\
\text { montrer ce qu'il faut modifier, et à montrer } \\
\text { comment modifier les choses. } \\
\text { - Du côté de l'élève, il sert à modifier la } \\
\text { production du son, à voir ce que montre le } \\
\text { professeur, et à entendre ce qui est joué. }\end{array}$ & $\begin{array}{l}\text { Contrat d'évaluation : l'élève interprète } \\
\text { les gestes du professeur, il s'évalue et } \\
\text { modifie ce qu'il fait à partir de ce qu'il } \\
\text { entend et des gestes du professeur. }\end{array}$ \\
\hline 6) entendre, sentir, ressentir & $\begin{array}{l}\text { Le milieu-soi de l'élève évolue : on y ajoute } \\
\text { la prise de conscience de la perception des } \\
\text { sensations corporelles, à un son correspond } \\
\text { une sensation corporelle au niveau du ventre } \\
\text { et des lèvres qui peuvent se substituer à l'ouie. }\end{array}$ & $\begin{array}{l}\text { Contrat de sensations (feeling ?): «Quand } \\
\text { tu joues en groupe, si tu ne t'entends pas, } \\
\text { recherche les sensations corporelles que tu } \\
\text { as identifiées quand tu jouais individuelle- } \\
\text { ment ce son." }\end{array}$ \\
\hline
\end{tabular}

Quatre exemples de ces jeux d'apprentissages sont détaillés dans la suite de l'article, extraits de la partie comprenant le travail instrumental en orchestre, qui renvoient aux types 3 à 6 du tableau 
didactique peut être accommodé par le milieu ainsi créé, et comment dans ce nouveau contrat le professeur et les élèves peuvent produire-déchiffrer de nouveaux signes, illustrant ainsi le processus d'équilibration didactique dans l'action conjointe.

\section{QUATRE MICRO-ANALYSES EMPIRIQUES}

Rectifier la stabilité d'une note produite collectivement à partir des indications du professeur

Dans cet extrait qui renvoie à un jeu d'apprentissage de type 3 du tableau, le professeur, après avoir fait jouer la note " $\mathrm{Mi}^{7}$ " à l'ensemble de l'orchestre, a isolé le pupitre des tubas pour le faire travailler en fonction de la difficulté. Il passe ensuite du pupitre à une élève en particulier, pour lui faire produire individuellement le son en fonction de la difficulté rencontrée. Une fois la difficulté résolue avec cette élève, il revient au pupitre pour une production collective du son. La rapidité de ces aller et retours entre collectif, pupitre et individus est facilement identifiable dans ses raisons pour un connaisseur qui entend les variations du son (justesse et timbre). Dans l'exemple que nous analysons, « Pg » demande aux quatre tubistes de rejouer ensemble un « mi ». Après leur avoir indiqué « respirez bien et soufflez, pincez vot'..., chut. », il accompagne, en silence et par sa gestuelle la production de leur son. Ce moment ne dure que quelques secondes, de 16'20 à 16'30, et il est illustré par les photogrammes du tableau 1.
Après avoir marqué la pulsation, le geste parallèle au sol illustre visuellement l'idée de tenir le son de façon continue en maintenant une même pression, comme si le souffle dessinait un trait.

La forme du geste en évoque une autre: on peut rapprocher celle de la représentation graphique de la fréquence des sons, dont l'amplitude est plus grande si le son est " grave » et se réduit au fur et à mesure qu'il devient aigu. La projection de l'air doit être rapide et linéaire, projection qui trouve une analogie dans le geste du bras (photogrammes 2). Ce geste représente aussi la tenue de la note dans le temps: à une projection de l'air suivie d'un son continu, correspond une projection du geste suivie d'un geste continu (photogramme 3 ).

Nous pouvons ainsi observer qu'ici, à une perception auditive correspond une représentation visuelle, qui est analogue dans sa forme à la constance qui est attendue dans la fréquence du son produit, et qui est représentée de la même façon, depuis le XIX ${ }^{\mathrm{e}}$ siècle. ${ }^{8}$

Le souffle est une sensation difficile à partager en tant que milieu, d'autant que les dimensions sonores de ce milieu sont déjà saturées par les sons produits par les tubistes. Le professeur est donc amené à créer un autre signe pour représenter une dimension du milieu, le souffle, sur lequel l'élève doit agir en assurant la meilleure constance possible. Le signe produit une analogie du souffle. Il permet ici à l'enseignant, d'attirer l'attention des élèves sur les éléments du milieu qui l'intéressent. Le milieu devient ainsi un «milieu-soi ». Le corps du professeur, par les mimes qu'il produit, est convoqué comme milieu ainsi que celui des élèves (vue et ouie), qui perçoivent celui du

Tableau 2 : le geste qui prolonge et complète la parole

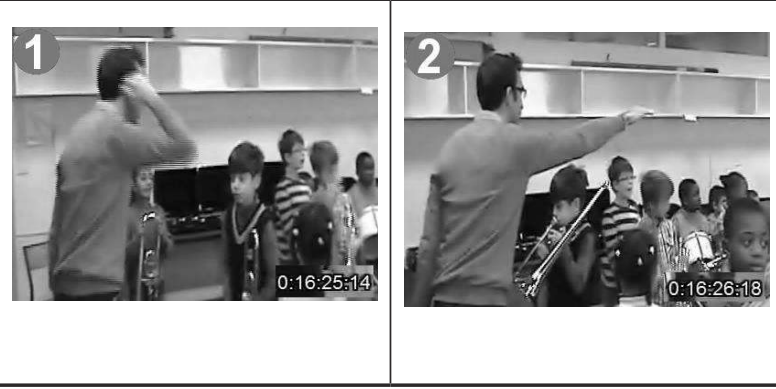

Le professeur regarde les élèves concernés. Il prolonge le geste classique de la battue en le transformant en un geste qui aura une autre signification. Sa main rejoint le niveau de l'oreille (photogrammel) ; elle est projetée par un mouvement horizontal et net du bras (photogramme 2) en direction des élèves tubistes, et elle se maintient ainsi pendant toute la durée de la production de leur son (photogramme 3).

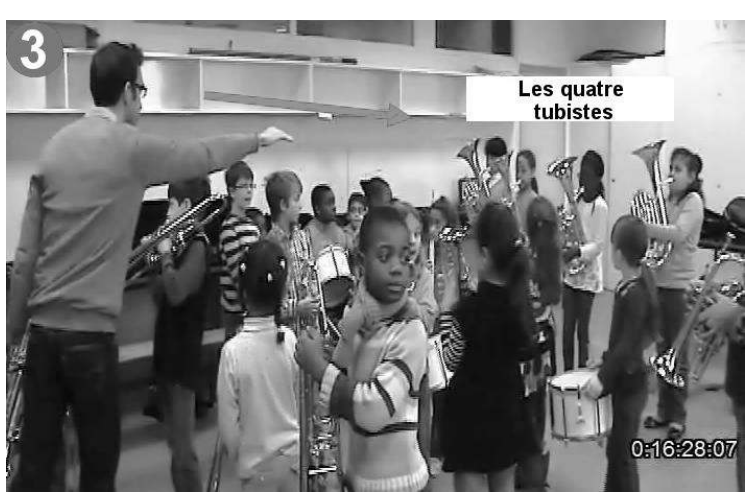


professeur et qui agissent sur la production de leur propre son. Le milieu fait donc ici appel à deux sens, l'ouie et la vue.

Le contrat didactique précédent (contrat d'imitation de la note produite par le professeur) évolue vers un contrat d'expérimentation (essai-erreur), du type : " imite ce que je fais, par analogie avec mes gestes, et rectifie au fur et à mesure de ce que tu entends et de ce que tu vois».

Ajuster le son en fonction du geste du professeur

Cet extrait renvoie à un jeu d'apprentissage de type 4 du tableau de synthèse. Le professeur a commencé l'étude d'une autre note, dans un prolongement rapide de la précédente (nous sommes à la minute 17 de la séance) : "On continue : le Fa. Un, deux, trois... ». À partir d'une configuration correspondant à un jeu d'apprentissage de type 2 , le professeur fait d'abord jouer à l'ensemble de l'orchestre un Fa, pendant qu'il le produit lui-même, avec sa trompette. S'intéressant alors au pupitre des trombones (17'15), il fait jouer successivement la même note par chaque trombone, en approuvant l'action des élèves (C'est bien,...). Puis il demande de nouveau (17'40) "les quatre trombones en même temps». Le professeur va être alors conduit, pendant la production sonore des élèves en petit collectif, à donner en cours de jeu des indications afin d'agir, non plus sur la constance, mais sur la hauteur du son produit.

Une fois lancée la production collective du son par les trombonistes, le professeur est en quelque sorte privé du recours à la parole, qui serait inaudible et viendrait perturber la production. Cette réalité est d'autant plus prégnante qu'il s'agit ici de produire un son de hauteur stable sur une durée significative (plusieurs secondes) : toute interruption de cette durée au motif de corrections verbales ou autres conduirait à perturber la nature même du jeu. Il s'agit donc de rendre les élèves attentifs à l'écoute du son qu'ils produisent. Ce que le professeur signale en montrant sa propre oreille, (photogramme 1) attirant ainsi l'attention sur un des sens, l'ouie et sur la nécessité de s'écouter. Mais cette condition une fois remplie, et le milieu « son » ainsi clairement désigné, il reste à donner des indications sur les attentes spécifiques à ce qui est produit, autrement dit à produire une sémiose qui permette aux élèves de s'orienter dans ce milieu sonore.

Cette condition est remplie par l'usage de l'index, qui après la désignation de l'oreille effectue à plusieurs reprises un mouvement de bas en haut (photogramme 2), indiquant clairement une nécessité d'ajuster le son vers le haut, c'est-à-dire vers

Tableau 3 : le geste qui remplace la parole

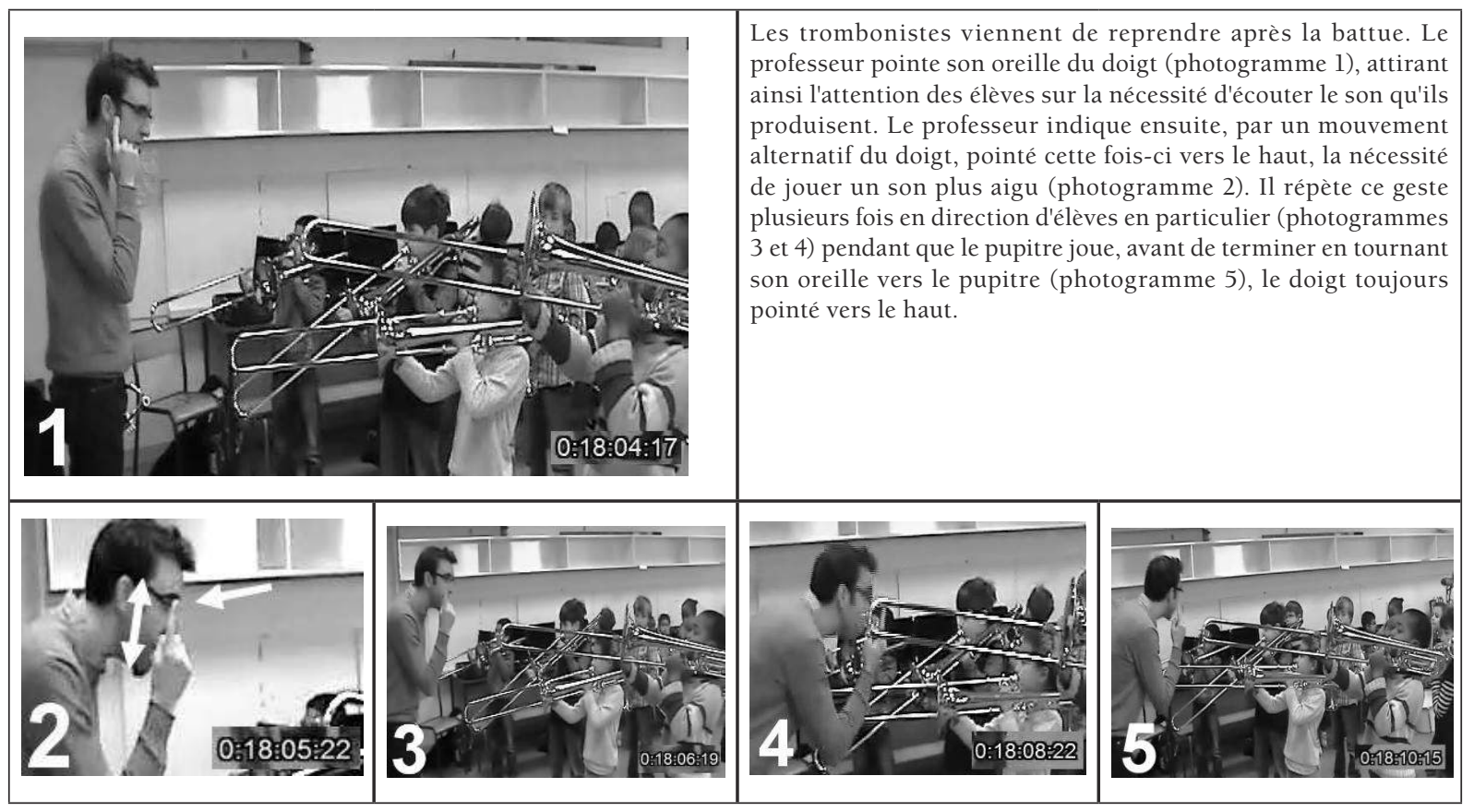


l'aigu (ici, le son). Cette répétition est accompagnée d'indications proxémiques (Forest, 2009), qui vont du regard appuyé (photogramme 3), jusqu'à des déplacements nettement visibles (photogramme 4) en direction de certains élèves en particulier à qui le signe «monter le son» est alors plus spécialement destiné. Cette sémiose du contrat concernant la hauteur du son se prolonge tout le long de la production, le retrait proxémique (photogramme 5) donnant à penser une attribution plus collective de la signification : si chaque élève doit jouer un son plus aigu, c'est bien au profit d'un son collectif, soit également plus aigu, comme l'indique la position de l'index, à la fois près de l'oreille et dirigé vers le haut.

On retrouve une explicitation verbale de l'analogie entre hauteur du son et représentation par le geste au moment où le professeur reprend l'étude du Fa, quelques instants plus tard, ainsi que le montre le tableau 4 ci-dessous. la connivence (méfiez-vous...), le «ça » évoquant une force contre laquelle il convient d'être prévenu. La responsabilité de l'élève est ainsi atténuée. Seule reste l'attention qui doit être collectivement portée à cette propension à tomber, d'un son qui est qualifié de " grave ", associant ainsi la hauteur du son dans le vocabulaire, et le niveau montré par le doigt ou ici, la main. L'analogie entre hauteur du son et hauteur de la main est renforcée : le son grave tombe. Déjà présente dans l'extrait précédent, elle pourra de nouveau être convoquée, autant que de besoin, en situation d'orchestre.

Le professeur a donc ici partagé une visualisation de la hauteur du son, plus aigu en haut, et plus grave en bas, que les élèves comprennent et ont vite intégrée. Ce nouveau contrat didactique pourrait être formulé ainsi : « si le son n'est pas juste, c'est qu'il est soit trop haut, soit trop bas et le(s) signe(s) du professeur en donne(nt) l'indication ». Le milieu dans ses

Tableau 4 : le geste et les mots pour le dire

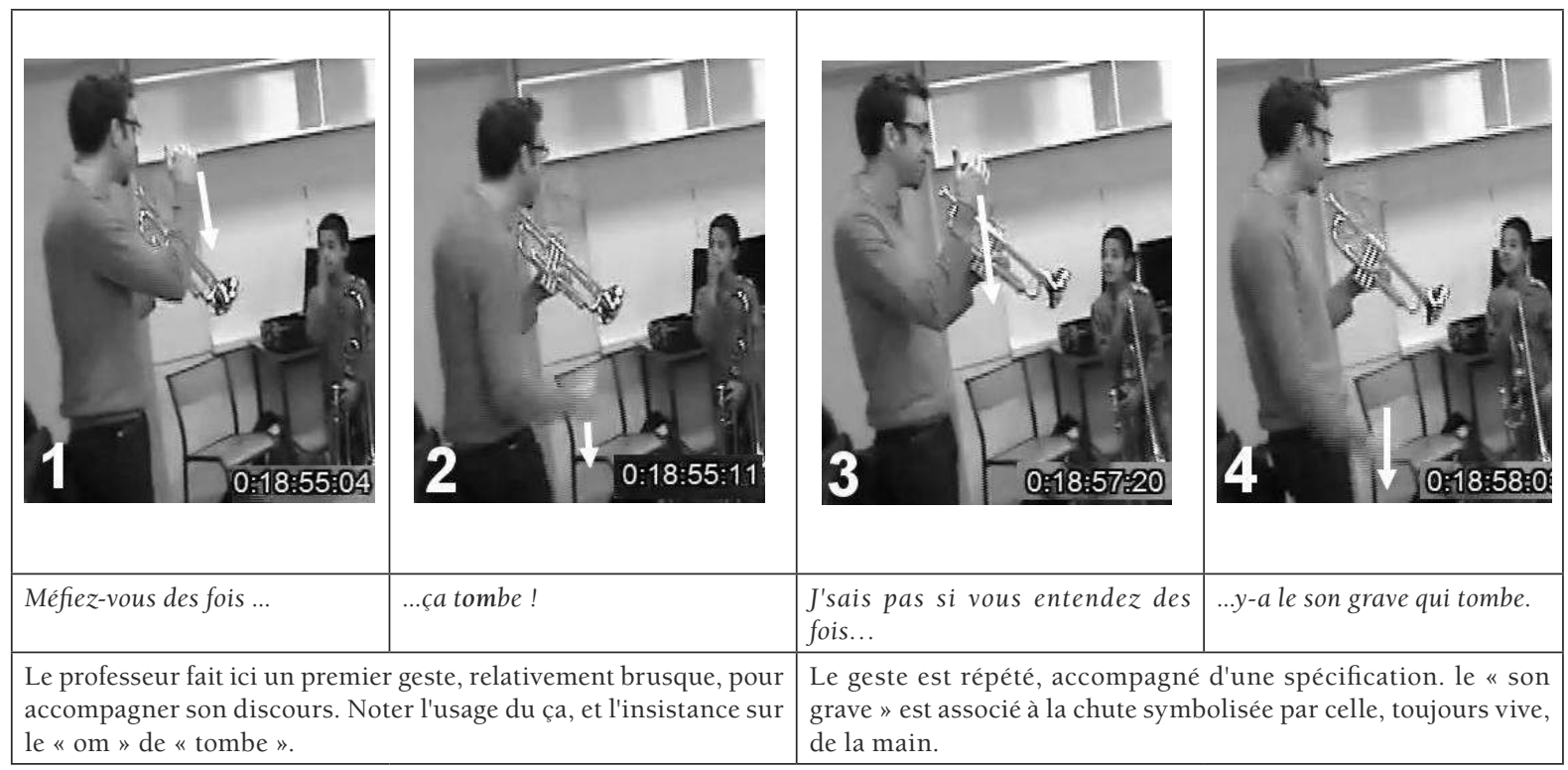

Cette explicitation verbale est rendue possible par la pause sonore entre le Fa, qui vient d'être étudié, et le Sol, dont l'étude va commencer juste après. Le professeur insiste sur la question qui vient déjà d'être traitée en situation (Cf tableau 3 précédent). Une nouvelle signification (symbolisée par la chute de la main) indique aux élèves une propension du son à « tomber » (ça tombe). L'énoncé du professeur, d'une certaine façon, est produit dans une position topogénétique assez basse, sur le mode de simples caractéristiques sonores n'est pas suffisant pour générer spontanément, par ses rétroactions, un ajustement des capacités des élèves. La gestuelle du professeur donne une information supplémentaire sur ses attentes en ajoutant à l'information sonore une information visuelle. Elle consiste en une représentation analogique du son. À la constance nécessaire du souffle, objet de l'équilibration précédente, est ajoutée la stabilité de la hauteur du son, représentée par les gestes du professeur. Du point de vue 
Tableau 5 : un geste pour un autre

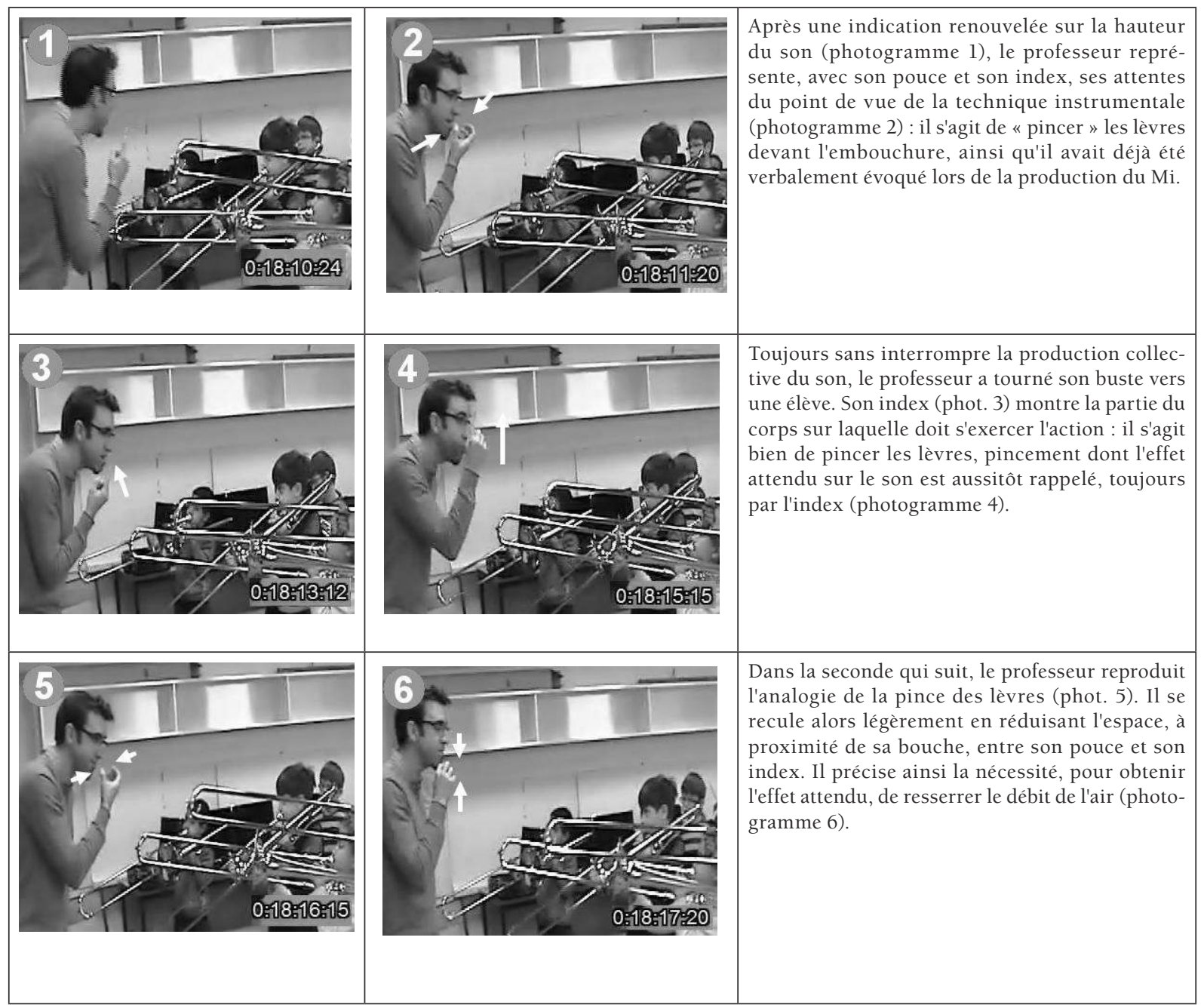

des élèves, le problème est ainsi reconfiguré pour leur permettre simultanément :

- la relation au son produit par l'enseignant (imitation du son préalablement produit)

- la relation au son produit par eux-mêmes (et comparaison éventuelle avec le précédent) - la relation aux gestes qui représentent les éléments sonores du milieu, dans une analogie avec ce qui est corporellement ressenti

Pour autant, la production d'un son suffisamment juste nécessite également l'accommodation d'une autre dimension contractuelle, liée à la maîtrise technico-corporelle de l'instrument. Pour ce faire, le professeur va introduire dans le milieu de nouvelles significations concernant cet aspect.
Établir un rapport entre le son, la justesse, la technique et le geste du professeur

Cet extrait renvoie à un jeu d'apprentissage de type 5. Nous avons vu dans l'exemple précédent que les élèves jouent et que, pour ne pas interrompre le son afin qu'il subsiste en tant que milieu, Pg donne des consignes de souffle et de justesse uniquement par gestes, sans exemple sonore ${ }^{9}$. Voyons maintenant comment le professeur réussit, quasi simultanément, à donner également des indications permettant l'accommodation de la technique instrumentale. Dans les photogrammes suivants ; tous les trombonistes sont en train de produire un fa, et l'on perçoit, à l'écoute; les variations de hauteur du son.

Un son aigu, produit avec un instrument à embouchure, demande physiquement au musicien 
une pression d'air produite par la bouche qui doit être fine et concentrée, pression obtenue en resserrant les lèvres. Plus le son est aigu, plus le débit de l'air doit être concentré et la projection rapide. L'émission de l'air doit être plus étroite et l'orifice par lequel il sort doit être plus petit et plus ferme (donc formé par une bouche dont les lèvres sont fermes et resserrées). C'est cette combinaison qui produit une vibration de l'air plus rapide (Ricquier, 1982).

Les photogrammes montrent comment le professeur représente, par des gestes, l'émission de l'air et la forme que doivent prendre les lèvres, dans le but de jouer un son plus aigu et donc plus juste. Ils sont reproduits plusieurs fois en fonction des besoins identifiés par le professeur pour informer les élèves de ce qu'il attend d'eux. L'écoute sur la vidéo du son produit révèle les modifications qu'apportent les élèves à leur production en réaction aux gestes du professeur.

L'alternance très rapide (de l'ordre de la seconde) entre les signes qui représentent des consignes de justesse et ceux portant sur la technique instrumentale, montre le lien très étroit qui existe entre les deux. Les élèves l'ont bien compris, puisque qu'on peut les entendre réagir également très rapidement. L'étude attentive de cette action conjointe, qui vise la résolution du problème de la justesse de la note, nous conduit à en préciser les dimensions transactionnelles.

Les gestes, tout d'abord, ne peuvent être considérés comme des solutions, car particulièrement en musique il n'est pas possible de faire « à la place de »; ils sont comme des indicateurs qui permettent à l'élève de savoir sur quel élément porte la modification qu'il doit apporter à son jeu ${ }^{10}$, pour gagner. C'est là la difficulté de l'analyse, où le geste modifie certes le milieu, en y introduisant des significations nouvelles, mais il est aussi une modification en soi $\mathrm{du}$ contrat didactique, par la dimension qu'il indique, dimension sur laquelle l'élève peut s'appuyer, sans pour autant avoir une solution tenue pour vraie.

L'échelle de temps (de l'ordre de la seconde) est également remarquable: de micro-contrats didactiques, spécifiés à un savoir précis (qui relève ici de la technique des instruments à vent) viennent très rapidement s'ajuster au contrat initial. Le milieu est ainsi modifié tout aussi rapidement. En conséquence, on a affaire à une succession de micro-jeux d'apprentissage, avec des micro-jeux épistémiques émergents tout aussi fins et spécifiques.
Dans cet extrait, l'attente du professeur est toujours que l'élève joue un son juste. Ne l'obtenant pas de façon assez précise avec les précédents contrats (ancrage au sol, imitation sonore, doigt levé pour indiquer la modification de justesse...), il produit un nouveau contrat qui peut être explicité comme suit : "fais techniquement ce que je te montre en regardant et en imitant avec ta bouche ces signes nouveaux $»$. Le milieu inclut les nouveaux signes du pouce et de l'index de la main droite, signes qui représentent la forme que doit avoir la bouche et les lèvres. Le corps est ainsi convoqué comme milieu, celui du professeur comme celui des élèves, et les parties sur lesquelles il faut agir sont précisées. Si on peut considérer qu'il s'agit d'un contrat d'imitation, il repose sur une relation aux signes de type métaphorique.

À ce stade de l'analyse, nous pouvons déjà distinguer, en première approximation un contrat d'imitation intrinsèque, corporel (stabiliser son corps à l'image de celui du professeur) ou sonore (produire le même son que celui produit par le professeur). Ces contrats sont complétés par un contrat d'imitation analogique de type 1 (imite dans ta production sonore la stabilité que je représente avec ma main), un contrat d'imitation analogique de type 2 (imite dans ta production sonore la modification de hauteur du son que je représente avec mon doigt pointé vers le haut), auxquels vient s'ajouter un contrat d'imitation métaphorique corporelle (du type faire avec ta bouche ce que je fais avec la main). Nous allons maintenant illustrer un dernier type de contrat, qui renvoie au corps de l'élève.

Intégrer la perception de son corps comme une référence du jeu instrumental dans la pratique collective : entendre, sentir, ressentir

Jusqu'à présent, nous avons vu que la perception auditive permettait une évaluation du son produit par l'élève. Elle référait à deux entités, le corps du professeur et celui de l'élève :

- Du côté du professeur, le corps sert à montrer ce qu'il faut modifier, et à montrer comment modifier les choses.

- Du côté de l'élève, il sert à modifier la production du son, à voir ce que montre le professeur, et à entendre ce qui est joué. 
Tableau 6 : le corps de l'élève comme signe

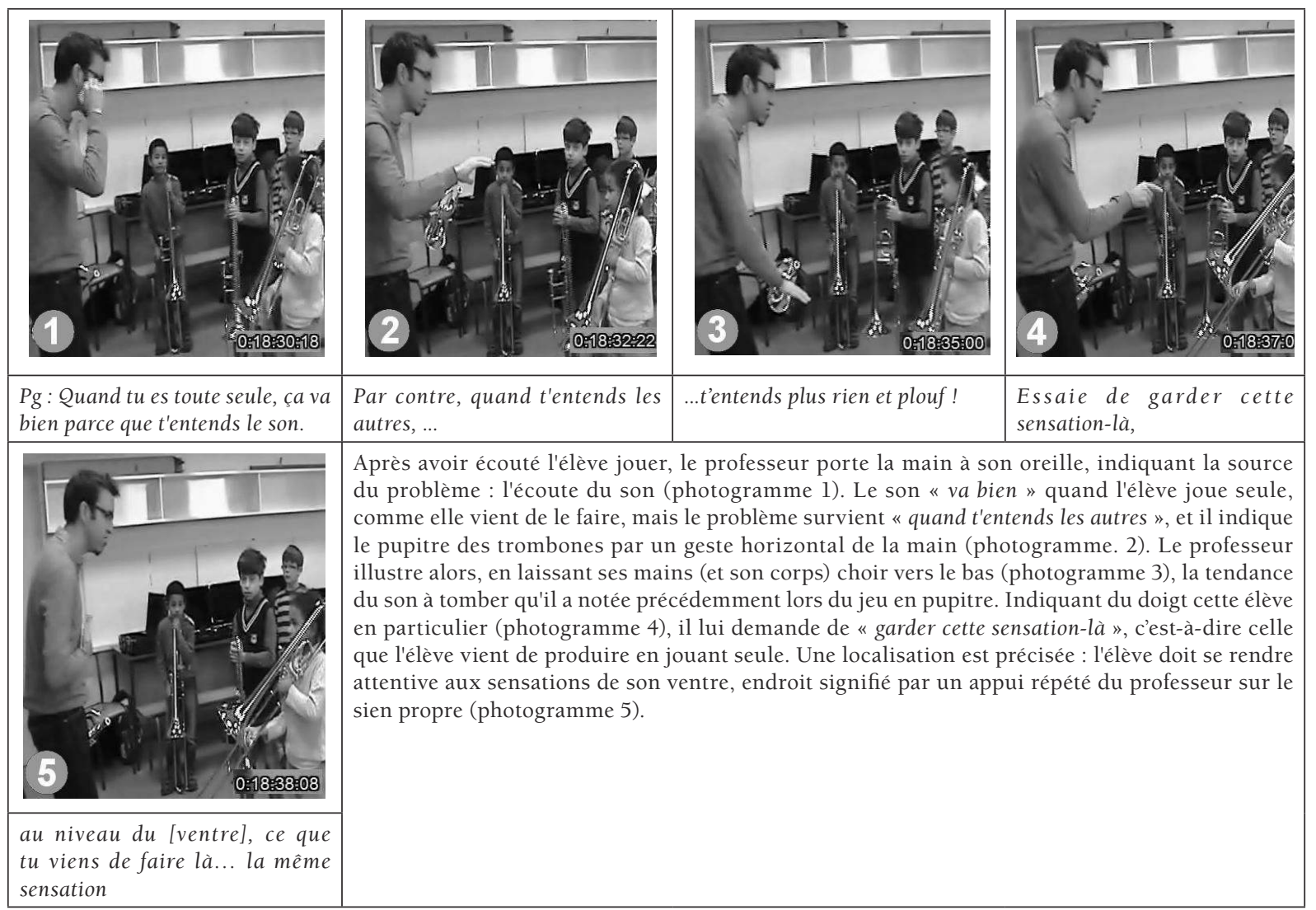

L'extrait qui suit se situe à la fin de la production collective du Fa, dont nous avons présenté l'analyse ci-dessus. La transaction porte sur les difficultés qu'a rencontrées une élève lors de la production collective. Le professeur lui demande d'abord de rejouer le Fa, seule, et il commente ensuite sa production sonore, satisfaisante quand elle joue seule, mais qui « tombe » quand elle joue avec les autres.

Le problème ici posé est lié à la difficulté, pour un musicien d'orchestre, d'ajuster sa propre production instrumentale en fonction de la hauteur du son qu'il produit, alors que la perception auditive de ce son est largement perturbée par les sons produits par les autres musiciens du même pupitre, et plus largement de l'orchestre.

La transaction signifie ici qu'à un son correspond une autre sensation, localisée par le professeur au niveau du ventre, et que cette sensation corporelle peut être un palliatif au problème d'audition multiple rencontré lors du jeu en orchestre. Le musicien, ici l'élève, doit pouvoir se rendre sensible à ces autres sensations que strictement sonores, pour avoir un retour sur le son qu'il produit lui-même, et l'ajuster aux autres sons entendus. Mais il faut pour cela que cette sensation ait été identifiée dans des conditions d'écoute favorable et donc à un moment de travail et d'apprentissage individuel.

Le professeur cherche ainsi à élargir les capacités de perception des élèves par rapport à un son, en cherchant à leur faire éprouver le fait (physique) qu'un son produit ne résonne pas seulement dans l'ouie (cf les jeux d'apprentissage de type 3 et 4 ), et ne vibre pas seulement au niveau des lèvres (cf le jeu d'apprentissage de type 5), mais a aussi des incidences dans d'autres parties du corps, le corps même de celui qui le produit. L'enjeu est donc tout d'abord de signaler ce fait (la sensation dans le « ventre » par un contrat métaphorique langagier) et sera ensuite de réussir à accorder cette perception personnelle à la perception du son ambiant pour que, à la fin, l'orchestre "sonne». Le milieu est ici étendu par le professeur au-delà des dimensions strictement 
sonores. Les rétroactions peuvent prendre leur source dans l'ensemble du corps, qui devient luimême élément de ce milieu.

On a ici un travail d'équilibration contrat-milieu qui n'est plus directement métaphorique, mais sensible. Le milieu (le son produit par l'élève) agit sur les sensations (perceptions des vibrations dans son corps) dans un premier temps puis les sensations (signalées et localisées au niveau du ventre par le professeur) participent au milieu-soi.

\section{RÉSULTATS ET DISCUSSION}

\section{De la spécificité de l'enseignement d'une pratique instrumentale en orchestre}

Les descriptions et analyses que nous avons produites montrent que la notion de milieu, dans le cas d'une pratique instrumentale en orchestre, nécessite d'être spécifiée. Nous développons ici trois dimensions de ce milieu, que nous avons respectivement nommé :

- le milieu-son, qui renvoie à la production effective des vibrations de l'air perçues par l'ouie,

- le milieu-corps, qui renvoie au corps comme support des techniques permettant un usage adéquat de l'instrument de musique, et - le milieu-soi, qui renvoie à l'usage du corps comme source de sensations, ce "feeling " permettant à la fois la justesse du son produit par l'instrumentiste, et son accord avec les sons produits par les autres.

Concernant la première dimension du milieu nous avons vu que le son, bien que perçu par les élèves, n'était pas spontanément considéré par eux dans les contraintes ni les rétroactions qu'il permet. L'identification des variations de ce milieu-son nécessite donc que le professeur, après avoir nommé le son dans le langage (en lui attribuant la note, par exemple le Fa), attire l'attention des élèves sur ses caractéristiques. Mais le son est par essence invisible, soumis à l'écoulement du temps, et rend évidemment difficile toute indication verbale pendant sa production. Certains aspects de la sémiose du contrat restent clairement identifiables (cf par exemple l'indication par le professeur de son oreille aux fins d'orienter l'attention des élèves vers l'écoute). Mais dans beaucoup de cas, le professeur est conduit à produire, en même temps qu'une indication contractuelle, une représentation gestuelle de ce milieu-son, que ce soit du point de vue de sa hauteur (Cf tableau 3, phot. 2, 3 et 4 et tableau 5, phot. 4) ou de sa stabilité (tableau 2). Ces indications gestuelles en cours de production bénéficient régulièrement d'une explicitation dans le langage, lors d'une pause dans le jeu (ça tombe, tableau 4 , phot. 2 et 4 ; et plouf!, tableau 6 phot. 3). L'apprentissage d'un instrument en orchestre suscite ainsi une double sémiose spécifique, car elle ne peut prendre directement appui sur les techniques d'orientation de l'attention couramment observées dans d'autres domaines d'enseignement (voir par exemple Forest \& Mercier, 2010 ; Forest, 2012), pas plus que sur des signes du milieu qui resteraient permanents après leur production (ibid.).

La deuxième dimension relève des techniques du corps dont l'apprentissage, selon Mauss (1936) prend appui sur « l'imitation prestigieuse ». Il s'agit, dans notre cas pour les élèves, de parvenir à utiliser un instrument d'une façon qui permette la production d'une variété de sons, choisis et constants. Les techniques utilisées pour ce faire présentent des aspects génériques, comme par exemple celles consistant à assurer à son corps un certain type de stabilité, ce qui peut être réalisé par un jeu d'apprentissage par imitation somme toute assez classique (cf le jeu d'apprentissage de type 1 du tableau 1 , non illustré dans cet article). Mais certaines techniques sont très spécifiques à l'instrument, ici un instrument à embouchure, et nécessitent l'adaptation tout aussi spécifique du corps de l'élève. S'agissant par exemple du pincement des lèvres, la technique est difficile à voir, et même à percevoir. Cet ensemble de contraintes conduit le professeur, pour rendre visible ce qu'il attend de l'élève, à représenter cette fois-ci une partie de son corps (les lèvres) par une autre (son pouce et son index), mimant ainsi pendant le jeu et avec ses doigts au bord des lèvres la technique labiale qu'il attend de l'élève en train de souffler dans son instrument.

Nous avons nommé la dernière dimension « milieu-soi », car il s'agit, pour l'élève, de se rendre sensible à des dimensions corporelles qui ne peuvent résulter de l'exemple du professeur. Les sensations vibratoires doivent être identifiées aux sensations sonores. Situées par les indications du professeur au niveau du ventre, elles relèvent d'une perception particulière. Il s'agit pour l'élève d'orienter son action sur son propre corps, ici de constituer les vibrations 
qui résonnent en lui comme sources de possibles rétroactions lui permettant d'ajuster la note qu'il produit, à défaut de pouvoir la distinguer par l'ouie parmi l'ensemble produit par l'orchestre. On remarquera que cette dernière dimension, du point de vue d'un connaisseur, s'exprimera plutôt dans un registre esthétique, en termes de "feeling».

On peut sans doute considérer cette dernière dimension comme fortement liée à la spécificité de la pratique instrumentale en orchestre. Mais sa mise en évidence peut aussi conduire à renouveler, en lien avec les deux précédentes, la question de la place du corps en tant que milieu dans les apprentissages.

\section{Sémiose didactique}

Nous avons constaté dans les analyses à quel point ces différentes dimensions du milieu sont étroitement imbriquées. De la même façon, il est parfois difficile de distinguer, dans le flux de la double sémiose didactique, les signes du milieu des signes contractuels, les deux étant souvent indissolublement liés.

Si l'on prend pour exemple la contraction des lèvres (tableau 5), on peut soutenir que les gestes du professeur relèvent d'une sémiose épistémique : le signe du pincement est directement lié au savoir jouer du trombone et à la forme de l'embouchure, corps et trombone étant ainsi constitués comme milieu. Mais, par sa proximité de ses doigts avec ses lèvres, et le transfert entre ce qui est attendu (le pincement des lèvres) et ce qui est produit (le pincement pouce-index), le professeur produit bien un signe à dimension contractuelle, indiquant à la fois une attente portant sur les lèvres de l'élève avec une orientation de l'attention vers celles-ci. On a ici une relation sémiotique de type métaphorique, que l'on peut paraphraser pour en montrer la complexité : «fais avec tes lèvres ce que je te montre avec mes doigts ».

On peut se demander si un tel enchevêtrement ne pourrait pas être également constaté dans tout apprentissage mettant en jeu un instrument (y compris des instruments autres que musicaux). Tout instrument est en effet porteur, de par sa forme, son origine et son histoire, d'un certain nombre d'attentes qui font, par exemple, qu'une trompette est quelque chose ou l'on souffle, un tambour ou un piano quelque-chose où l'on tape (et un marteau quelque chose avec lequel on tape sur quelque chose). Mais il suffit d'essayer de souffler dans une trompette ou de jouer du piano (ou d'enfoncer un clou de charpentier avec un marteau) pour s'apercevoir de la résistance que ces mêmes instruments, en tant que milieu, opposent à l'action.

Quoiqu'il en soit, cet enchevêtrement temporellement très fin des différentes sémioses rend nécessaire, pour leur mise au jour, une étude tout aussi détaillée qui convoque toutes les dimensions, corporelles et verbales de l'action. D'un point de vue méthodologique, seul un agencement comprenant photogrammes, énoncés et commentaires peut rendre visible cette finesse des transactions. Un tel agencement dans un article de revue reste toutefois incomplet compte tenu, s'agissant de musique, de la dimension effectivement sonore du milieu. On pourra s'en convaincre en regardant attentivement le moment rapporté dans le tableau 5 : seule l'écoute des variations du son pourrait effectivement attester de la sémiose réciproque professeur-élèves, et de la façon dont la variation du son suscite les gestes du professeur qui entraînent eux-mêmes la remontée plus ou moins collective du son, entraînant de nouveau des gestes, etc. Un système de publication multimédia qui donnerait à entendre et voir simultanément ces éléments de façon convaincante, serait sans doute ici nécessaire.

\section{Dialectique milieu-contrat, et équilibration didactique}

L'étude de cette séance permet d'observer comment, dans le déroulement du temps didactique, chaque milieu contient en germe une accommodation potentielle du contrat antérieur, elle-même indispensable à l'assimilation d'une part du milieu suivant, assimilation partielle qui permet la prise en compte d'autres rétroactions générant elle-même une accommodation, etc. C'est bien parce que les élèves ont été rendus capables d'identifier (et de produire par imitation) un Mi que le professeur peut donner des indications sur la technique de projection du son (tableau 2) ou sur sa hauteur (tableaux 3 et 4). Cette conscience de la hauteur et de la constance est ellemême utile pour que les élèves puissent éprouver les variations qu'ils pourront produire en suivant les indications du professeur sur l'action des lèvres (tableau 5). De la même façon, ce n'est que sur la 
base d'une production individuelle raisonnablement stable et juste que le professeur peut ensuite insister sur la nécessité de prendre en compte les sensations corporelles pour maintenir dans le collectif la stabilité qui a pu être obtenue individuellement (tableau 6).

Cet « emboîtement » épistémique dans les jeux d'apprentissage illustre un certain type de relation entre un " temps d'objet » et un " temps de situation », décrit par ailleurs par Sensevy (2011, p. 592) à propos des ingénieries didactiques. La progression ici adoptée par le professeur n'est pas simplement consécutive : chaque objet spécifique étudié, par exemple le pincement des lèvres pour produire une note stable et juste, n'est pas étudié simplement pour lui-même. Il est systématiquement étudié en référence aux objets antérieurement rencontrés (stabilité du corps, justesse de la note), et il n'est traité qu'en rapport avec une situation : faire sonner collectivement l'orchestre. Chaque technique enseignée, chaque régulation proposée prend son origine dans l'activité individuelle et collective des élèves. Et c'est la situation qui renvoie, lorsque les élèves jouent, les significations susceptibles d'être exploitées par le professeur et perçues dans leur sens culturel original.

On peut s'interroger sur la présence d'un tel type de relation entre temps d'objet et temps de situation dans une séance qui n'est pas issue d'une démarche d'ingénierie. Dans cette séance comme dans la plupart de celles observées à l'occasion de cette recherche, on peut en outre constater l'attention soutenue et prolongée des élèves alors que beaucoup d'entre eux sont considérés comme peu susceptibles d'un tel effort d'attention et de travail prolongé. Pour amorcer la réflexion sur ce point, nous souhaiterions attirer, en conclusion, l'attention sur un commentaire du professeur dans le tableau 4 , sur le son qui « tombe », et sur l'analyse que nous en avons produite. On constate en effet que le professeur, par son discours (méfiez-vous, ça tombe) se place dans une position topogénétique relativement basse. En leur demandant de se "méfier», le professeur indique aux élèves une difficulté profonde de la pratique à laquelle tout praticien doit faire face, y compris le professeur (même si, lui, l'a résolue). La valeur relativement neutre du «ça » permet en outre d'insister sur l'idée qu'il s'agit d'une contrainte de la « chose musicale », et non de la conséquence d'une carence des élèves. On est donc bien loin de la correction d'un comportement inadéquat, type de transaction à laquelle ces élèves peuvent par ailleurs être habitués : il s'agit de produire un résultat tous ensemble contre la nature résistante du milieu, résultat dont chacun, dans un orchestre, est comptable. Car les élèves ne sont pas totalement ignorants de la chose musicale, et ils sont capables d'éprouver dans une certaine mesure la qualité sonore de ce résultat.

L'enseignement d'une pratique instrumentale en orchestre, menée comme on l'a vu, pourrait ainsi conduire à une expérience authentique de la puissance des savoirs. Une telle expérience est d'autant plus importante pour des élèves qui n'en sont pas familiers, lorsqu'elle débouche sur une émotion esthétique partagée à laquelle chacun a pu participer. 


\section{NOTES}

1. Notons que P (que nous nommons également «le professeur ») ou E (« l'élève ») doivent être considérés comme des instances, c'est-à-dire qu'ils peuvent représenter suivant les situations une ou plusieurs personnes.

2. On trouvera une première approche des spécificités du film d'étude, et en particulier sur la différence analogiquedigital dans Forest, 2006, 2008, Forest \& Mercier 2010 et un développement plus complet sur la question du film d'étude en didactique dans Sensevy, 2011, dans le chapitre 6. Ce chapitre est disponible en ligne à l'adresse suivante: http://python.bretagne.iufm.fr/sensevy/sensdusavoir/ LeSensDuSavoirChap6.pdf

3. Pour un développement sur la nature et les raisons de ces techniques phénoménographiques, voir le chapitre déjà cité ci-dessus.

4. le non verbal en musique comprend des signes musicaux produits (les sons) qui ne permettent pas au professeur d'être entendu. Un autre mode de communication non verbal est donc nécessaire, ici les gestes du professeur, vecteurs privilégiés des attentes du-dit professeur et que nous décrirons dans l'analyse.

5. Un son peut être décrit par sa fréquence: le la3 à $440 \mathrm{~Hz}$, ou à $4442 \mathrm{~Hz}$ selon le choix des instrumentistes. Jouer juste serait alors s'approcher au plus près d'une fréquence définie au préalable. Cependant si la justesse est un prérequis dans le jeu d'expert, elle reste toujours relative. Nous pouvons, par exemple, nous souvenir des moments très expressifs de la Callas, d'Edith Piaf, d'Ivry Gytlis, chez lesquels une légère fêlure dans la voix ou dans le jeu, nous fait vibrer. Ici le jeu d'apprentissage est bien la justesse, mais son degré d'acquisition varie en fonction de l'enseignant et/ou du niveau dans lequel l'élève se trouve à un moment « $\mathrm{T} »$. Le propos n'est donc pas de savoir si les élèves jouent justes, mais comment fait le professeur pour obtenir, de la part des élèves, la justesse qu'il s'est définit lui-même.

6. Définir ce qu'est « jouer ensemble » demanderait une analyse très poussée et donc trop longue pour l'objet présent. Nous nous en tiendrons ici à la définition d'Arnold (1993) : «La réalisation d'un bon ensemble suppose de renoncer aux individualités au profit de la fusion globale des instruments ou des voix» (p. 719).

7. Les élèves apprennent le nom de la note en même temps qu'ils apprennent à la jouer. Mais n'ont pas encore abordé la lecture. Ils ne l'aborderont qu'au bout d'un an de pratique.

8. En lisant le traité d'instrumentation et d'orchestration et plus particulièrement Le chef d'orchestre. Théorie de son art de Berlioz (1843/2002), on s'aperçoit que ce sont les mêmes consignes gestuelles de base qui perdurent actuellement.

9. Dans cette séance, le professeur donne à chaque fois un exemple sonore de la note attendue avec sa trompette. Mais le sujet ici est de montrer comment ensuite, sans pouvoir utiliser le recours direct au son et compte tenu des contraintes qui s'exercent sur la parole, il arrive malgré tout à créer et modifier des éléments du milieu.

10. Le mot jeu est ici employé dans un double sens, celui du musicien et celui de la modélisation de la TACD. 


\section{RÉFÉRENCES}

Arnold, D. (1993). Dictionnaire encyclopédique de la musique (Vol. II). (M.-S. Pâris, Trad.) Paris : Robert Laffont.

Batézat-Batellier, P. (2012). Un apprentissage particulier dans une école élémentaire : l'instrument de musique en orchestre comme exemple d'une approche didactique de la musique. Mémoire de master de l'université de Rennes 2.

Chevallard, Y. (1992). Concepts fondamentaux de la didactique: perspectives apportées par une approche anthropologique. Recherches en didactique des mathématiques, 12 (1), 73-112.

Chevallard Y. (1996). La fonction professorale : esquisse d'un modèle didactique. In R. Noirfalise \& M.-J. Perrin-Glorian (Éds), Actes de la VIIIe école d'été de didactique des mathématiques (p. 83-122). ClermontFerrand, France : IREM.

Chauviré, C. \& Sackur, J. (2003). Le vocabulaire de Wittgenstein. Paris : Ellipses.

Forest, D. (2006). "Analyse proxémique d'interactions didactiques". Carrefour de l'Education, 21, 73-94

Filliettaz, L. (2005). Discours, travail et polyfocalisation de l'action. In L. Filliettaz et J.-P.. Bronckart, (Ed.), L'analyse des actions et des discours en situation de travail. Concepts, méthodes et applications (p.155-175). Louvain-La-Neuve : Peeters

Forest, D. (2008). Agencements didactiques, pour une analyse fonctionnelle du comportement non-verbal du professeur. Revue française de pédagogie, 165, 77-89.

Forest, D. (2012). Lattention conjointe dans le jeu didactique : une approche proxémique. In B. Gruson, D. Forest \& M. Loquet (Éds.), Jeux de savoirs, études de l'action conjointe en didactique. Rennes: PUR

Forest, D., Mercier, A. (2010). Vidéos de séances en classe et ressources d'enseignement, éléments d'analyse. In G. Gueudet, L. Trouche (Éds.), Ressources vives, le travail documentaire des professeurs, le cas des mathématiques (p. 321-339). Rennes / Lyon : Presses Universitaires de Rennes / Institut National de Recherche Pédagogique.

Foucault, M. (1963). Naissance de la clinique. Paris : PUF.

Ginzburg, C. (1989). Traces. Racines d'un paradigme indiciaire. In Mythes, Emblèmes, Traces. Morphologie et histoire, p. 139-180. Paris : Flammarion.
Glock, H. J. (2003). Dictionnaire Wittgenstein. Paris : Gallimard.

Goody, J. (1979). La Raison graphique. La domestication de la pensée sauvage. Paris: Minuit.

Gruson, B., Forest, D., \& Loquet, M. (2012). Jeux de savoirs, études de l'action conjointe en didactique. Rennes : PUR

Leutenegger, F. (2000). Construction d'une clinique pour le didactique. Une étude des phénomènes temporels de l'enseignement. Recherches en didactique des mathématiques, 20/2, 209-250.

Mauss, M. (1936/1997). Les techniques du corps. in Sociologie et Anthropologie, p. 365-386. Paris : PUF

Mondada, L. (2004). Temporalité, séquentialité et multimodalité au fondement de l'organisation de l'interaction : Le pointage comme pratique de prise du tour. Cahiers de Linguistique Française, 26, 169-192.

Peirce, C. S. (1998). The essential Peirce: (1893-1913). Bloomington : Indiana University Press.

Ricquier, M. (1982). traité méthodique de pédagogie instrumentale. Principe de la colonne d'air, respiration, maîtrise de soi, décontraction, relaxation. Paris : Gérard Billaudot.

Sensevy, G. (2011). Le sens du savoir. Éléments pour une théorie de l'action conjointe en didactique. Bruxelles : De Boeck

Sensevy, G. (2012). Le jeu comme modèle de l'activité humaine et comme modèle en théorie de l'action conjointe en didactique, quelques remarques. SPSS, 7-2, 105-133

Sensevy, G. \& Forest, D. (2012, July). Semiosis process in instructional practices. International Conference of the Learning Sciences, Sydney, Australia.

Sensevy, G., \& Quilio, S. (2002). Les discours du professeur : vers une pragmatique didactique. Revue Française de Pédagogie, 141, 47-56.

Sensevy, G., \& Mercier, A. (2007). Agir ensemble, l'action didactique conjointe du professeur et des élèves. Rennes : PUR.

Veyne, P. (1971). Comment on écrit l'histoire. Paris : Le Seuil. 